\title{
Transition of Morphologic Features in Lupus Nephritis: Does Steroid Therapy Accelerate Glomerulosclerosis?
}

\author{
Hirofumi MaKino, Yasushi Yamasaki, Kenichi ShIKata, \\ Naoki Kashihara, Hitoshi Sugryama, Toshio Ogura and Zensuke Ota
}

\begin{abstract}
We retrospectively evaluated the morphologic change of 28 follow up biopsies from 24 cases of lupus nephritis according to the classification of the World Health Organization and determined the activity index (AI) and the chronicity index (CI). In the cases with biopsies repeated within 6 months, the AI decreased significantly from $6.7 \pm 1.3$ to $3.5 \pm 0.8$, while the CI showed no significant change. In cases which were rebiopsied after longer intervals, AI increased significantly from 3.2 \pm 0.7 to 7.5 \pm 1.2 ; the $C I$ did not change significantly. When AI and CI changes in the cases biopsied again beyond 6 months were compared with respect to therapy, AI showed no significant difference in the methylprednisolone pulse therapy group but was significantly increased in the oral steroid therapy group. The CI tended to be increased in both groups, but not significantly. Steroid pulse therapy was effective in improving active lesions with a high AI. Steroid therapy for lupus nephritis prevented short-term progression of glomerulosclerosis and did not accelerate glomerulosclerosis. (Internal Medicine 34: 982-987, 1995)
\end{abstract}

Key words: systemic lupus erythematosus, glomerulonephritis, steroid hormone, therapy

\section{Introduction}

Lupus nephritis is caused by deposition of immune complexes in the glomerulus as a result of aberrant immunologic activity (1). Since lupus nephritis is extremely heterogeneous both clinically and pathologically, pathologic assessment by renal biopsy is very important for the establishment of prognosis and for the selection of appropriate therapy (2-7). For histological evaluation, the World Health Organization (WHO) classification scheme (8) is used widely. This approach defines not only the histologic morphology but also establishes the degree of activity and sclerosis of a given lesion (8).

An Italian group followed 659 patients with lupus nephritis for ten years and reported that hypertension and diffuse proliferative glomerulonephritis (WHO class IV) is associated with a worse prognosis (9). Nossent et al (10) reported that clinical tests of renal function were not reliable in discriminating between active lesions and chronic renal damage. However a chronicity index of $>3$ was associated with decreased patient survival. Esdaile et al (11) and McLaughlin et al (12) also reported the importance of the chronicity index for the prediction of prognosis, especially in cases with normal renal function (12).
It is known that the glomerular lesions of lupus nephritis may change with disease progression or as a result of treatment (4, 13). It has also been reported that steroid therapy actually may accelerate glomerulosclerosis (14). We previously reported the effect of pulse methylprednisolone therapy on lupus nephritis as defined by the WHO morphologic classification (15). We also reported the clinical significance of necroses in response to steroid therapy (16). In the present study, we retrospectively reviewed morphologic changes including active and sclerosing lesions in lupus nephritis. Patients who underwent repeated biopsies were divided into one of two groups: pulse methylprednisolone therapy group and oral steroid group.

\section{Subjects and Methods}

\section{Patients}

The subjects enrolled in this study consisted of 24 patients with lupus nephritis who underwent serial renal biopsies and met four or more criteria of the Preliminary Criteria for the Classification of Systemic Lupus Erythematosus (17) of the American Rheumatoid Association for cases treated prior to 1982 and the 1982 Revised Criteria for Systemic Lupus Erythematosus (18) for the cases treated thereafter.

From the Third Department of Internal Medicine, Okayama University Medical School, Okayama

Received for publication February 6, 1995; Accepted for publication July 11, 1995

Reprint requests should be addressed to Dr. Hirofumi Makino, the Third Department of Internal Medicine, Okayama University Medical School, 2-5-1 Shikatacho, Okayama 700 


\section{Histological classification, activity and chronicity indices}

Renal tissues were studied by light microscopy, immunofluorescent microscopy, or electron microscopy for classification according to the WHO method. Activity as well as chronicity of renal lesions was scored according to the method of Austin et al (19). The activity index (AI) was based on the extent of glomerular cell proliferation, karyorrhexis, cellular crescent formation, and interstitial mononuclear cell infiltration and scored as 1 (mild or less than 25\%), 2 (moderate or from 25\% to less than $50 \%$ ), or 3 (severe or $50 \%$ or more). The presence of karyorrhexis and cellular crescents, which are reflective of active lesions, doubled the above scores, and 2 points each were added when wire-loops, hematoxyphil bodies, or fibrin thrombi were present. Similarly, increased mesangial matrix, glomerular sclerosis, and tubular and interstitial lesions were scored as $0-3$, respectively which were also added to the total CI score. The AI and CI score were determined by three nephrologists without prior knowledge of the source of the specimen.

\section{Analysis of histological changes after steroid therapies}

Histological changes in lupus nephritis were studied with respect to $\mathrm{AI}, \mathrm{CI}$, and therapy used in the 24 cases with follow up biopsies. The interval between biopsies ranged from 2 months to 7 years. Two cases involved three biopsies and two cases involved four biopsies. Sixteen of the 24 cases were treated with pulse methylprednisolone therapy and 8 were treated with oral steroid therapy. The third and fourth biopsies also were included in the study. Two biopsies were excluded from the study, because the specimen for light microscopy did not contain enough glomeruli to calculate AI and CI. Thus, a total of 28 biopsies were evaluated in this study. These cases treated with methylprednisolone pulse therapy after repeated biopsy were counted in the pulse group, and cases treated with only conventional oral prednisolone were included in the oral steroid group. The serial biopsy cases were classified according to whether the follow-up biopsy was earlier than or later than 6 months after the initial biopsy. Because the effect of pulse methylprednisolone therapy is usually judged after 2 to 6 months and rebiopsy was performed on patients manifesting the exacerbation of renal symptoms such as an increase in proteinuria. The short-term group consisted of 3 cases treated with oral therapy and 12 cases treated with pulse therapy. The long-term group consisted of 8 cases treated with oral therapy and 5 cases treated with pulse therapy. AI and CI were compared between groups.

\section{Protocol of steroid therapies}

The protocol of steroids therapy used was the same as in our previous studies $(15,16)$. Pulse therapy consisted of methylprednisolone, $1,000 \mathrm{mg}$, dissolved in $500 \mathrm{ml}$ of $5 \%$ glucose solution and administered by intravenous infusion over 2 hours for 3 consecutive days. This was followed by oral doses of 30 $40 \mathrm{mg} / \mathrm{day}$. Oral steroid therapy consisted of prednisolone, initiated at $30-50 \mathrm{mg} / \mathrm{day}$ and then tapered. In both groups the initial dose of prednisolone was administered for 4 to 8 weeks and then decreased according to the immunoserological activi- ties such as CH50 and anti-DNA antibodies. The decision to choose conventional oral steroid therapy or pulse methylprednisolone therapy was at the discretion of the patient together with his or her physician. None of the cases received immunosuppressants such as cyclophosphamide, azathioprin, cyclosporin A or mizoribin.

\section{Statistical analysis}

Data are presented as mean \pm SEM. Statistical analysis was performed by Wilcoxon test. A level of $\mathrm{p}<0.05$ was considered as statistically significant.

\section{Results}

\section{Morphologic transition after steroid therapies}

Twenty-eight follow-up biopsies in 24 cases revealed morphologic transitions in 14 cases, all of which involved class IV lesions except for one case: 3 changed from class IV to class V, 3 from III to IV, and 2 each from II to IV and vice versa; two improved, one changing from IV to I, one from III to I, and 2 worsened from class IV to class VI (Fig. 1).

Repeated biopsies showed that, among class III lesions, one case treated with pulse therapy improved to class I, and 3 cases treated with oral therapy progressed to class IV. In 8 class IVa cases, 1 case each improved to class I or class II, 2 worsened to class IV-b, 1 transformed to class V, and 3 were unchanged. In 5 class IVb cases with severe active nephritis, 2 evolved to IVc, 1 case each evolved to class V, class VI, or unchanged. Of the 3 cases in class IVc with active and sclerosing nephritis, 1 case each progressed to class IVd, class V, and class VI (Fig. 2).

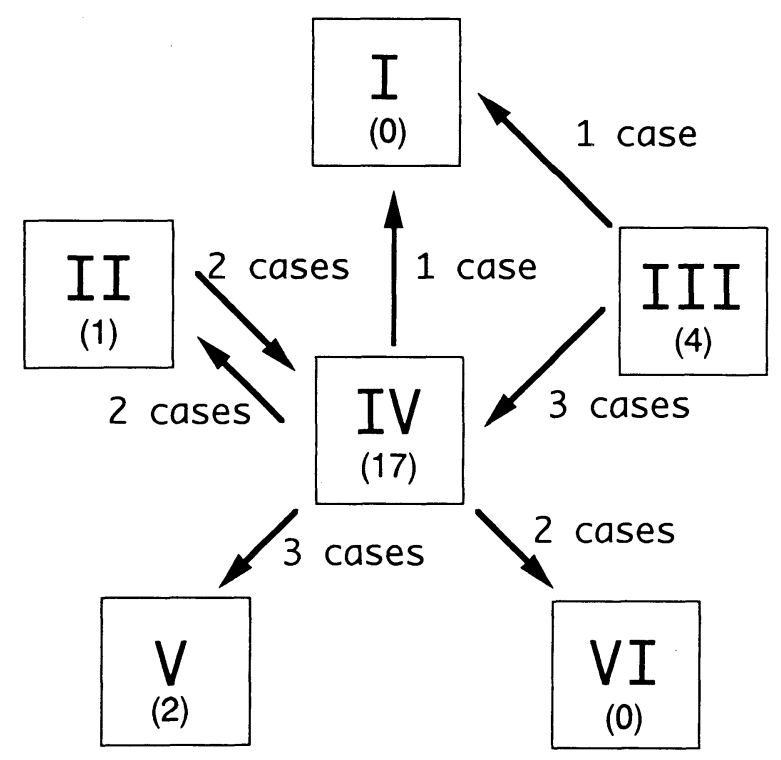

Figure 1. Transition of the morphological classification of lupus nephritis from the first biopsy to the last biopsy. ( ) indicates number of cases diagnosed at first biopsy. 
Changes of activity and chronicity indices after steroid therapies

In order to study the effects of treatment on active and sclerosing lesions, the cases with follow-up biopsies were divided into a short-term group (repeat biopsy within 6 months) and a long-term group for comparison of the $\mathrm{AI}$ and $\mathrm{CI}$. In the short-term group, which consisted of 3 cases treated with oral therapy and 12 cases treated with pulse therapy ( 15 cases in total), the AI decreased significantly from a pretreatment mean of $6.7 \pm 1.3$ ( \pm SEM) to $3.5 \pm 0.8(\mathrm{p}<0.01)$. There was no signifi-

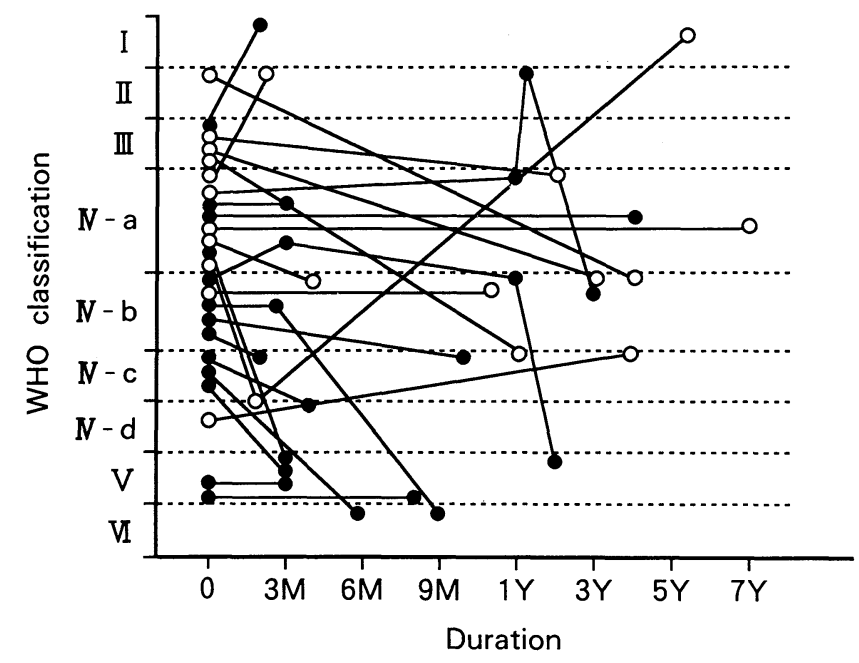

Figure 2. Time-course of the transition of morphological classification in lupus nephritis. $\bullet-\bullet$ pulse therapy,,$-\circ$ conventional oral prednisolone therapy. cant change in CI, which was $1.7 \pm 0.7$ before treatment and $1.6 \pm 0.4$ after treatment. In the long-term group, which consisted of 8 cases treated with oral therapy and 5 cases treated with pulse therapy ( 13 cases in total), the AI increased significantly from $3.2 \pm 0.7$ to $7.5 \pm 1.2$, while the $\mathrm{CI}$ also increased, but not significantly, from $1.1 \pm 0.4$ to $1.7 \pm 0.4$ (Fig. 3). When these indices were compared between the oral and parenteral treatment groups within the long-term group, the AI tended to increase, though not significantly, from $2.8 \pm 1.2$ to $6.2 \pm 1.8$ in the pulse therapy group, but it increased significantly in the conventional oral therapy group from $3.5 \pm 0.8$ to $8.4 \pm 1.6$ $(\mathrm{p}<0.05)$. The CI tended to increase both in the pulse (from $1.0 \pm 0.3$ to $1.4 \pm 0.7$ ) and the oral therapy groups (from $1.1 \pm 0.6$ to $1.9 \pm 0.5$ ), but the increase was not significant (Fig. 4).

\section{Discussion}

Morphologic transitions in lupus nephritis may result from the natural history of the disease or from treatment $(4,13,14$, 20). The most frequent transition is from class III to class IV, as Silva (20) reported in 27 of 66 cases and Baldwin et al (4) found in 27 of 151 cases. In the present study, 3 of 5 cases with repeat biopsies after one year or longer had progressive disease on the follow-up biopsy. Balow et al (14) have reported that 38 of 237 cases (16\%) progressed from classes II or III to IV, 10 of 420 (2.4\%) shifted from class II, III or IV to class V and 4 of 55 cases (7\%) shifted from class V to class III or IV. Here we found no transitions from class $\mathrm{V}$ to other classes, but transitions from class II, III, or IV to class V were seen in 3 of 22 cases (13.6\%). The transitions noted shortly after pulse therapy were likely due to the selective response of glomerular subendothelial immune deposits. This implies that the mechanism of production of

\section{a) Short term group}

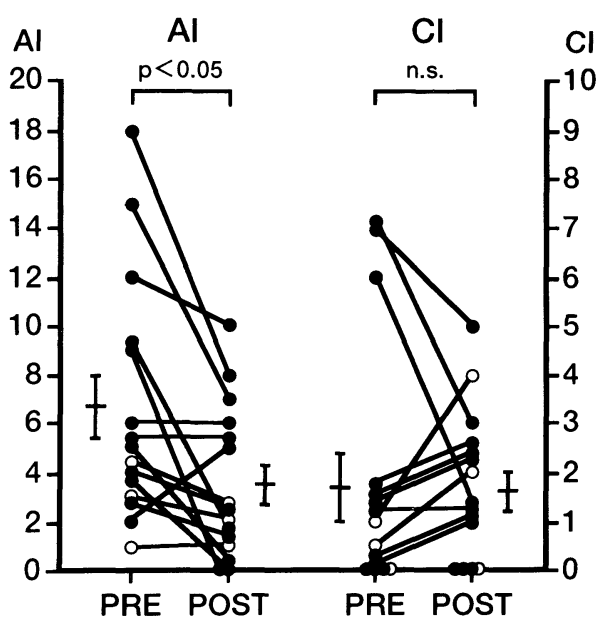

\section{b) Long term group}

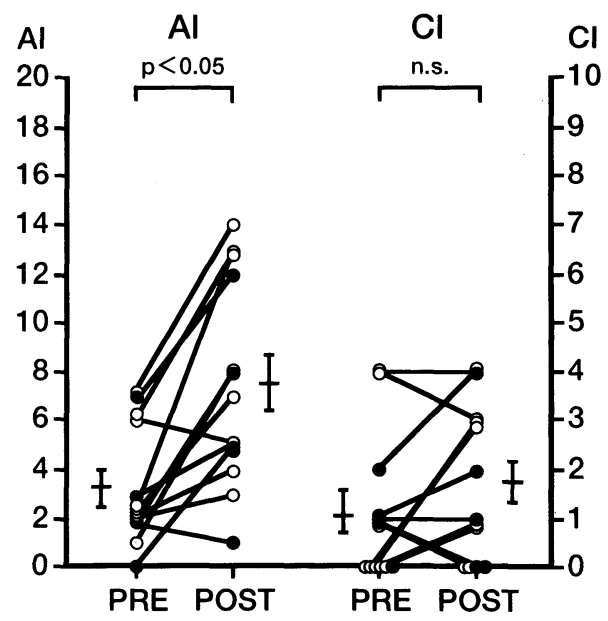

Figure 3. Changes in the activity and chronicity indices detected by repeated renal biopsies conducted at intervals within 6 months (A) and beyond 6 months (B). $\bullet$ pulse therapy, $\multimap$ conventional oral prednisolone therapy. AI: activity index, CI: chronicity index. 
(A) Changes of Activity Indices

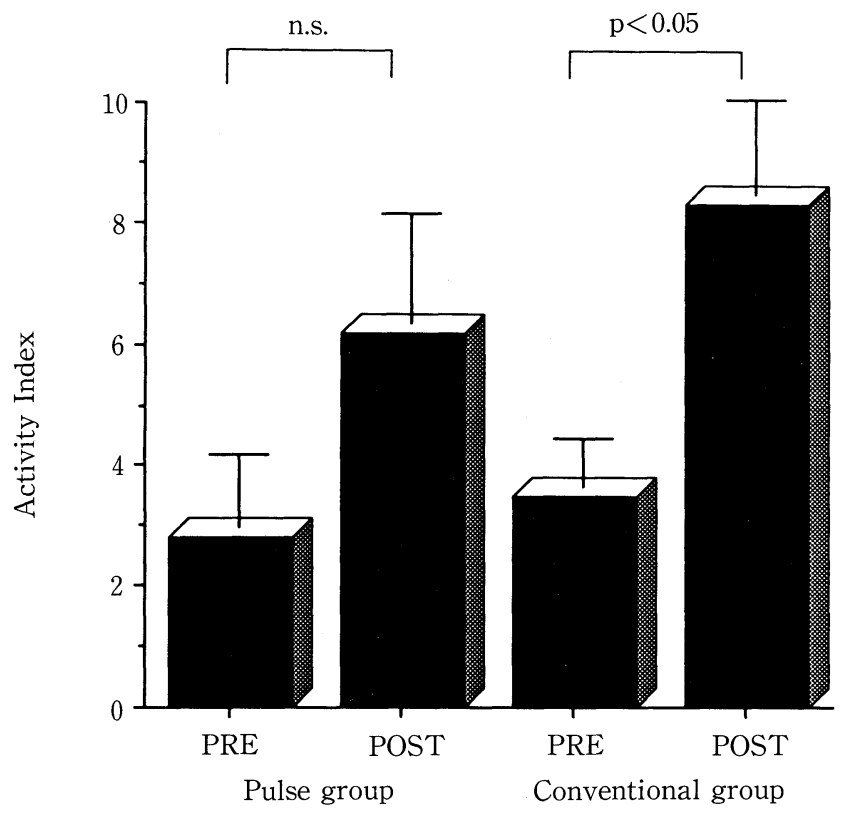

(B) Changes of Chronicity Indices

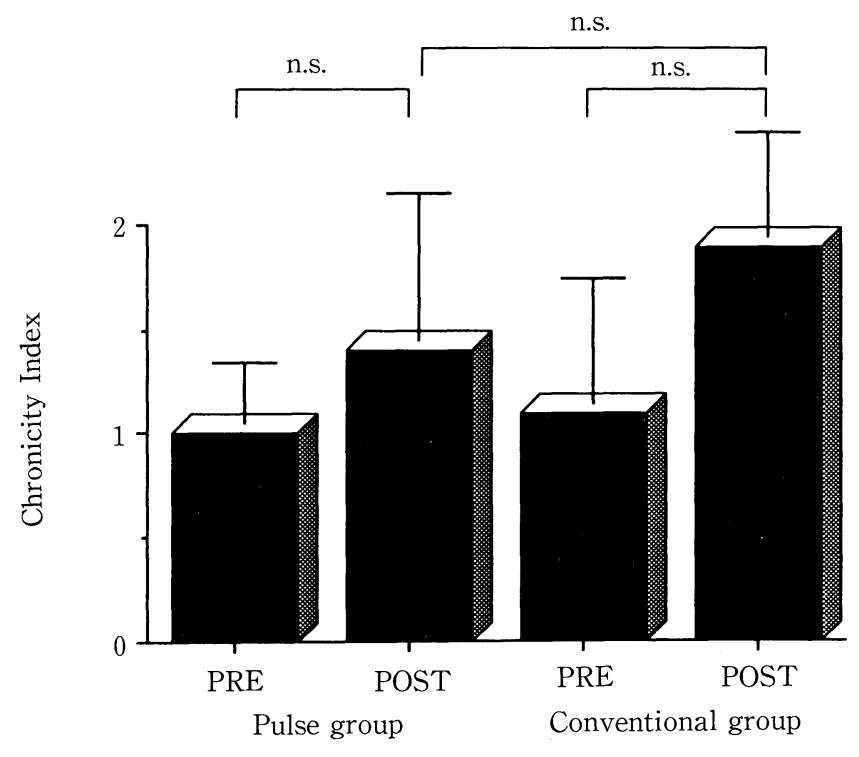

Each bar represents mean \pm SEM

Figure 4. Comparison between the activity index and chronicity index of initial and repeated renal biopsies performed beyond 6 months in both the methylprednisolone pulse group and the oral prednisolone group.

immune complexes differs among classes III, IV, and V. Some monoclonal anti-DNA antibodies have been found to react with immune deposits in class IV but not with those in class $\mathrm{V}$ disease (21).

The standard therapy for lupus nephritis is primarily ster- oids. However, disease progression cannot always be controlled with steroid therapy alone. The use of steroids over a long period also has the potential problem of accelerating glomerulosclerosis. Balow et al (14) retrospectively studied the effects of steroids alone or in combination with cytotoxic drugs such as cyclophosphamide and azathioprine on lupus nephritis. Biopsies performed after an average interval of 44 months revealed an increased $\mathrm{CI}$ in those receiving steroids alone but not in the group on combination therapy. It was thus concluded that prednisolone alone cannot fully control nephritis or inhibit the progression of glomerular damage to sclerosis. They further reported the beneficial effect of combination therapy of lowdose prednisone with intravenous cyclophosphamide compared to oral prednisone alone (22). Steinberg and Steinberg (23), also reported the long-term benefit of cyclophosphamide with prednisone on active lupus nephritis. However, a more recent study indicates an increase in chronicity 5 years after intravenous cyclophosphamide treatment (24). Donadio and Glassock critically reviewed the effect of immunosuppressive drugs and questioned their long-term effects per se on lupus nephritis (25). They speculated that the favorable effects of immunosuppressive drugs were due to lower prednisone dosage and better medical management of hypertension, hyperlipidemia, infection and other metabolic consequences of systemic lupus erythematosus.

Okada (26) examined renal biopsy specimens taken from 13 cases of lupus nephritis before and after methylprednisolone pulse therapy in the context of AI and CI scores (27). AI decreased markedly from 9.2 to 4.3 , as in the present study, but $\mathrm{CI}$ increased from 0.8 to 1.3 overall, and from 0.8 to 1.6 in class IV. From these results, it was concluded that high-dose steroid therapy controls severe histologic damage but not sclerosis.

Short-term exposure to steroids increases renal blood flow (28). Steroids also, however, increase blood pressure through $\mathrm{Na}$ retention, vasoconstriction, and increased renin substrate. Steroids also accelerate arteriosclerosis through altered lipid metabolism or by direct effects on blood vessels and suppression of renal prostaglandin production (29). These effects may affect renal function when steroids are used over a long period.

In lupus nephritis, the major site of damage is the glomerulus due to immune complex deposition, although tubulointerstitial and vascular lesions are important. Interstitial fibrosis and tubular atrophy are also secondarily caused by the glomerular damage. Thus CI well reflects glomerulosclerosis. The glomerular matrix is composed of type IV collagen (30), laminin, heparan sulfate proteoglycan (31), and fibronectin, etc. In cases of various types of glomerulonephritis with glomerulosclerosis, increases in these matrix components have been reported (32). It is interesting to consider the manner by which steroids effect the production of matrix components. Kasinath et al (33) cultured glomerular epithelial cells and reported that dexamethasone increases the production of the heparan sulfate proteoglycan core protein. However, the regulation of type IV collagen production and other components in the glomerulus and the possible effects of steroids have not been fully elucidated. Transforming growth factor (TGF)- $\beta$ has some effect on 
the production of extracellular matrix and plays a role in various types of nephritis $(34,35)$. Influence of steroids on TGF- $\beta$ and other cytokines is also interesting.

Repeat biopsies in our study demonstrated a markedly decreased AI in the short-term group. In cases with activity indices of 9 or greater, the effect of pulse therapy was striking. There were no significant changes in CI. A few cases showed a decrease in $\mathrm{CI}$ in the short-term pulse group. This might be due to the variability of glomerular damage among glomeruli in lupus nephritis. In the long-term cases, many of which had repeat biopsies after recurrence of clinical nephritis, the AI increased significantly but the CI showed only a statistically insignificant tendency to increase. This tendency was similar in the pulse and oral therapy groups, and increases in both $\mathrm{AI}$ and $\mathrm{CI}$ were greater in the oral therapy group, though not statistically significant for $\mathrm{CI}$. In the short-term group, pulse therapy seemed to be effective in improving the AI. CI did not increase in this group and steroids were unlikely to cause sclerosis in the short term. Although the therapeutic effect was more difficult to evaluate in the long-term group, which included cases of recurrent nephritis and unresponsive cases, the more marked increase in the $\mathrm{AI}$ and the greater upward tendency of $\mathrm{CI}$ in the oral steroid group demonstrated that pulse therapy did not accelerate glomerulosclerosis more than oral therapy. It is more likely that ineffective control of lupus nephritis may have accelerated glomerulosclerosis. The addition of cytotoxic drugs such as cyclophosphamide might be beneficial in the long term.

Although it has been reported that steroids used over a long period may accelerate sclerosis (14), we found no such effect over a 6-month period. The pulse therapy was especially effective in improving active lesions, and methylprednisolone pulse therapy was beneficial for cases with high activity indices.

\section{References}

1) Fournie GJ. Circulating DNA and lupus nephritis. Kidney Int 33: 487, 1988.

2) Pollak VE, Pirani CL, Schwartz FD. The natural history of the renal manifestations of systemic lupus erythematosus. J Lab Clin Med 63: 537, 1964.

3) Hill GS, Hinglais N, Tron F, Back JF. Systemic lupus erythematosus. Morphologic correlations with immunologic and clinical data at the time of biopsy. Am J Med 64: 61, 1978.

4) Baldwin DS, Gluck MC, Lowenstein J, Gallo GR. Lupus nephritis: clinical course as related to morphologic forms and their transitions. Am J Med 62: 12, 1977.

5) Pillemer SR, Austin III H, Tsokos GT, Balow JE. Lupus nephritis: Association between serology and renal biopsy measures. J Rheumatol 15: $284,1988$.

6) Appel GB, Cohen DJ, Pirani CL, Meltzer JI, Estes D. Long-term follow up of patients with lupus nephritis. A study based on the classification of the world health organization. Am J Med 83: 877,1987.

7) McLaughlin J, Gladman DD, Urowitz MB, Bombardier C, Farewell VT, Cole E. Kidney biopsy in systemic lupus erythematosus. II. Survival analysis according to biopsy results. Arthritis Rheum 34: 1268, 1991.

8) Churg J, Sobin LH. Renal Disease. Classification and Atlas of Glomerular Disease. Igaku-Shoin, Tokyo, 1987, pp.127.

9) Gruppo Italino per lo Studio dell Nefrite Lupica (GISNEL). Lupus nephritis: Prognostic factors and probability of maintaining lifesupporating renal function 10 years after diagnosis. Am J Kidney Dis 19:
473,1992

10) Nossent HC, Henzen-Logmans SC, Vroom TM, Berden JHM, Swaak TJG. Contribution of renal biopsy data in predicting outcome in lupus nephritis. Analysis of 116 patients. Arthritis Rheum 33: 970, 1990.

11) Esdaile JM, Abrahamowicz M, MacKenzie T, Hayslett JP, Kashgarian M. The time-dependence of long-term prediction in lupus nephritis. Arthritis Rheum 37: 359, 1994.

12) McLaughlin J, Bombardier C, Farewell VT, Gladman DD, Urowitz MB. Kidney biopsy in systemic lupus erythematosus. II. Survival analysis controlling for clinical and laboratory variables. Arthritis Rheum 37: 559, 1994.

13) Baldwin DS. Clinical usefulness of the morphological classification of lupus nephritis. Am J Kidney Dis 11: 142, 1982.

14) Balow JB, Austin III HA, Muenz LR, et al. Effect of treatment on the evolution of renal abnormalities in lupus nephritis. N Engl J Med 311: $491,1984$.

15) Makino H, Yamasaki Y, Hayashi Y, et al. Effect of methylprednisolone pulse therapy in patients with lupus nephritis assessed by WHO morphologic classification. Jpn J Nephrol 32: 791 , 1990 (abstract in English).

16) Makino $H$, Hayashi $Y$, Yamasaki $Y$, et al. Clinical significance of necrosis in lupus nephritis. Int Med 33: 461, 1994.

17) Cohen AS, Reynolds WE, Franklin EC, et al. Preliminary criteria for the classification of systemic lupus erythematosus. Bull Rheum Dis 21: 643, 1971.

18) Tan EM, Cohen AS, Fries J, et al. The 1982 revised criteria for systemic lupus erythematosus. Arthritis Rheum 25: 1271, 1982.

19) Austin III HA, Muenz LR, Joyce KM, Antonovych TT, Balow JE. Diffuse proliferative lupus nephritis: Identification of specific pathologic features affecting renal outcome. Kidney Int 25: 689, 1984.

20) Silva FG. The nephropathies of systemic lupus erythematosus. in: Contemporary Issues in Surgical Pathology, Pathology of Glomerular Disease. Rosen S, Ed. Churchill Livingston, New York, 1983, vol. 1, p.79.

21) Sasaki T, Muroi T, Hatakeyama A, et al. Circulating anti-DNA immune complexes in active lupus nephritis. Am J Med 91: 355, 1991.

22) Austin HA III, Klippel JH, Balow JE, et al. Therapy of lupus nephritis. Controlled trial of prednisone and cytotoxic drugs. NEng J Med 314: 614, 1986.

23) Steinberg AD, Steinberg SC. Long-term preservation of renal function in patients with lupus nephritis receiving treatment that includes cyclophosphamide versus those treated with prednisone only. Arthritis Rheum 34: 945, 1991.

24) Valeri A, Radhakrishan J, Estes D, et al. Intravenous pulse cyclophosphamide treatment of severe lupus nephritis: a prospective five-year study. Clin Nephrol 42: 71, 1994.

25) Donadio JV, Glassock RJ. Immunosuppressive therapy in lupus nephritis. Am J Kidney Dis 21: 239, 1993.

26) Okada M. Methylprednisolone pulse therapy; a histological evaluation. Kidney Dialysis 8: 535, 1980 (in Japanese).

27) Pirani CL, Salinas-Madrigal L. Evaluation of percutaneous renal biopsy. Pathol Ann 3: 249, 1968.

28) Bermudez L, Hayslett JP. Effect of methylprednisolone on renal function and the zonal distribution of blood flow in the rat. Circ Res 31: 44, 1972.

29) Ichikawa Y. Does large dose of glucocorticoid accelerate renal insufficiency in lupus nephritis? Sogorinsho 37: 2772, 1988 (in Japanese).

30) Kefalides NA. Isolation of a collagen from basement membranes containing three identical $\alpha$ chains. Biochem Biophys Res Commun 45: 226, 1971.

31) Makino H, Gibbons M, Reddy MK, Kanwar YS. Nephritogenicity of antibodies to proteoglycans of the glomerular basement membrane-1. J Clin Invest 77: 142, 1986.

32) Nishimura S, Makino H, OtaZ. Three-dimensional ultrastructural changes of acellular glomerular basement membrane in various types of human glomerulonephritis. Nephron 53: 9, 1989.

33) Kasinath BS, Singh AK, Kanwar YS, Lewis E: Dexamethasone increases heparan sulfate proteoglycan core protein content of glomerular epithelial cells. J Lab Clin Med 115: 196, 1990. 


\section{Transition of Lupus Nephritis}

34) Border WA, Okuda S, Languino LR, Sporn MB, Ruoslahti E. Suppression of experimental glomerulonephritis by antiserum against transforming growth factor- $\beta$. Nature 346: 371, 1990.
35) Border WA, Okuda S, Languino LR, Ruoslahti E. Transforming growth factor $-\beta$ regulates production of proteoglycans by mesangial cells. Kidney Int 37: 689, 1990. 